ARTICLE

Received 20 May 2014 | Accepted 15 Jul 2014 | Published 19 Aug 2014

DOI: $10.1038 /$ ncomms5710

OPEN

\title{
Toll-like receptor 4 and MAIR-II/CLM-4/LMIR2 immunoreceptor regulate VLA-4-mediated inflammatory monocyte migration
}

Naoya Totsuka², Yun-Gi Kim¹,3,4, Kazumasa Kanemaru1, Kouta Niizuma1, Eiji Umemoto5, Kei Nagai, Satoko Tahara-Hanaoka1,6, Chigusa Nakahasi-Oda1, Shin-ichiro Honda1,3, Masayuki Miyasaka7, Kazuko Shibuya ${ }^{1} \&$ Akira Shibuya, ${ }^{1,3,6}$

Inflammatory monocytes play an important role in host defense against infections. However, the regulatory mechanisms of transmigration into infected tissue are not yet completely understood. Here we show that mice deficient in MAIR-II (also called CLM-4 or LMIR2) are more susceptible to caecal ligation and puncture (CLP)-induced peritonitis than wild-type (WT) mice. Adoptive transfer of inflammatory monocytes from WT mice, but not from MAIR-II, TLR4 or MyD88-deficient mice, significantly improves survival of MAIR-II-deficient mice after CLP. Migration of inflammatory monocytes into the peritoneal cavity after CLP, which is dependent on VLA-4, is impaired in above mutant and FcR $\gamma$ chain-deficient mice. Lipopolysaccharide stimulation induces association of MAIR-II with FcR $\gamma$ chain and Syk, leading to enhancement of VLA-4-mediated adhesion to VCAM-1. These results indicate that activation of MAIR-II/FCR $\gamma$ chain by TLR4/MyD88-mediated signalling is essential for the transmigration of inflammatory monocytes from the blood to sites of infection mediated by VLA-4.

\footnotetext{
${ }^{1}$ Department of Immunology, Faculty of Medicine, University of Tsukuba, 1-1-1 Tennodai, Tsukuba, Ibaraki 305-8575, Japan. ${ }^{2}$ Department of Immunology, Fukushima Medical University, 1 Hikarigaoka, Fukushima, Fukushima 960-1295, Japan. ${ }^{3}$ Japan Science and Technology Agency, Core Research for Evolutional Science and Technology (CREST), University of Tsukuba, 1-1-1 Tennodai, Tsukuba, Ibaraki 305-8575, Japan. ${ }^{4}$ Department of Pathology and Comprehensive Cancer Center, University of Michigan Medical School, 1500 East Medical Center Dr-4111 CCGC, Ann Arbor, Michigan 48109, USA. ${ }^{5}$ Laboratory of Immune Regulation, Department of Microbiology and Immunology, Osaka University, 1-1 Yamadaoka, Suita, Osaka 565-0871, Japan. ${ }^{6}$ Life Science Center of Tsukuba Advanced Research Alliance (TARA), University of Tsukuba, 1-1-1 Tennodai, Tsukuba, Ibaraki 305-8575, Japan. ${ }^{7}$ Interdisciplinary Program for Biomedical Sciences, Institute for Academic Initiatives, Osaka University, 1-1 Yamadaoka, Suita, Osaka 565-0871, Japan. Correspondence and requests for materials should be addressed to A.S. (email: ashibuya@md.tsukuba.ac.jp).
} 
$\mathrm{M}$ onocytes are able to differentiate into macrophages or dendritic cells to induce innate and adaptive immunities ${ }^{1}$. There are two subsets of murine monocytes: $\mathrm{CX}_{3} \mathrm{CR} 1^{\text {int }} \mathrm{CCR}^{+}{ }^{+}$Ly6C ${ }^{\text {high }}$ inflammatory monocytes (iMo), and $\mathrm{CX}_{3} \mathrm{CR} 1^{\text {high }} \mathrm{CCR}^{-}$Ly6Clow patrolling monocytes ${ }^{2,3}$. Monocyte recruitment is a crucial part of the host defense against invading microorganisms ${ }^{4}$. iMo are rapidly recruited to sites of infection and inflammation, where they protect the host from infection by bacteria, parasites or viruses ${ }^{5-7}$; however, they are also involved in the exacerbation of the pathogeneses of myocardial infarction, atherosclerosis and cancer ${ }^{8-10}$. iMo play an important role in protection from caecal ligation and puncture (CLP)-induced peritonitis, which is widely regarded as the most representative animal model of human polymicrobial sepsis ${ }^{11}$. iMo egress from the bone marrow into the peripheral blood via CC-chemokine receptor 2 (CCR2)-dependent mechanisms ${ }^{6,12}$. However, the recruitment of iMo from the blood to sites of infection is CCR2-independent; instead, it requires adhesion molecules such as very late antigen-4 (VLA-4) ${ }^{13,14}$. Conformational changes in individual integrin heterodimers leads to an increase in ligand-binding energy and a marked decrease in the rate of ligand dissociation ${ }^{15}$. Despite this knowledge, exactly how iMo transmigrate to sites of infection is not yet completely understood.

Myeloid-associated immunoglobulin-like receptor (MAIR)-II ${ }^{16-18}$ (also called LMIR2 (ref. 19) or CLM-4 (ref. 20)), which is encoded by $A F 251705$, is a member of the CD300 family of cell surface molecules that is encoded by nine genes on the small segment of mouse chromosome 11 (refs 20,21). The human CD300 family comprises seven members, which are encoded by genes located on chromosome 17 in a region syntenic to mouse chromosome 11 (ref. 22). MAIR-II is expressed on macrophages and a subset of $\mathrm{B}$ cells in the spleen and the peritoneal cavity ${ }^{16,17}$. MAIR-II noncovalently associates with the signalling adaptor DAP12, which bears the immunoreceptor tyrosine-based activating motif (ITAM), in macrophages and $\mathrm{B}$ cells ${ }^{16,18}$. MAIR-II mediates an activating signal for the production of the proinflammatory cytokines tumour necrosis factor (TNF)- $\alpha$ and interleukin (IL)- 6 in macrophages ${ }^{16,17}$. Interestingly, lipopolysaccharide (LPS) enhances FceRI $\gamma$ (FcR $\gamma$ chain) expression and induces the association of MAIR-II with $\mathrm{FcR} \gamma$ chain in peritoneal macrophages, but not in splenic macrophages or B cells, for which the lysine residue in the transmembrane region of MAIR-II is responsible ${ }^{17}$. In contrast, MAIR-II negatively regulates adaptive immune responses by $\mathrm{B}$ cells in a DAP12-dependent manner ${ }^{18}$. The physiological role of MAIR-II is not yet completely understood.

Here we show that MAIR-II is expressed on iMo and is involved in Toll-like receptor-4 (TLR4)-mediated iMo transmigration from the blood to sites of infection as part of the host response to polymicrobial peritonitis.

\section{Results}

AF251705 ${ }^{-/-}$mice are susceptible to peritonitis. To address the role of MAIR-II in myeloid cell function in vivo, we first investigated whether MAIR-II deficiency affected the pathophysiology of peritonitis in the CLP model. Wild-type (WT) mice that underwent CLP exhibited $<10 \%$ mortality at 7 days after CLP, whereas the AF251705 ${ }^{-1-}$ mice showed markedly shorter survival than WT mice (Fig. 1a and Supplementary Fig. 1). Severe multiorgan injury was observed in $A F 251705^{-/}$mice but not in WT mice (Fig. 1b). The number of peritoneal aerobic bacteria was increased in AF251705 $-1-$ mice compared with WT mice at $20 \mathrm{~h}$ after CLP (Fig. 1c). Moreover, serum levels of TNF- $\alpha$ and IL-6 were significantly increased in $A F 251705^{-/-}$mice compared with WT mice after CLP (Fig. 1d). These results indicate that AF251705 ${ }^{-1-}$ mice are more susceptible to CLP-induced peritonitis than WT mice.

MAIR-II regulates the migration of M\&phage/Mo after CLP. As acute septic peritonitis promotes the recruitment of macrophages/monocytes $(\mathrm{M} \phi / \mathrm{Mo})$ and neutrophils to the peritoneal cavity $^{23,24}$, we next assessed the influx of $\mathrm{M} \phi / \mathrm{Mo}$ and neutrophils in the peritoneal cavities of WT and $A F 251705^{-1-}$ mice after CLP. Before CLP, WT mice and AF251705-I- mice did not show any difference in myeloid cell populations in the peripheral blood and bone marrow (Supplementary Table 1). Total cell number, the number of neutrophils $\left(\mathrm{CD} 11 \mathrm{~b}^{+} \mathrm{F} 4 / 80^{-}\right.$Ly6G $\left.{ }^{\text {high }}\right)$ and apoptotic cells $\left(\mathrm{PI}^{+}\right)$in the peritoneal cavities were increased in both WT mice and AF251705 $-1-$ mice 8 and $20 \mathrm{~h}$ after CLP. However, the number of $\mathrm{M} \phi / \mathrm{Mo}\left(\mathrm{CD} 11 \mathrm{~b}^{+} \mathrm{F} 4 / 80^{+}\right)$was significantly increased only in WT mice (Fig. 2a,b).

To examine the importance of the influx of $\mathrm{M} \phi / \mathrm{Mo}$ into the peritoneal cavity in the survival after CLP, we used liposomeencapsulated dichloromethylene bisphosphonate $\left(\mathrm{Cl}_{2} \mathrm{MBP}\right.$-liposomes) to deplete $\mathrm{M} \phi / \mathrm{Mo}$ in the CLP model. We administered liposome-encapsulated $\mathrm{PBS}$-liposomes or $\mathrm{Cl}_{2} \mathrm{MBP}$-liposomes to WT or AF251705 ${ }^{-1-}$ mice 1 day before CLP. At $20 \mathrm{~h}$ after CLP, $\mathrm{Cl}_{2} \mathrm{MBP}$-liposomes had selectively depleted $\mathrm{M} \phi / \mathrm{Mo}$, but not neutrophils, in the peritoneal cavity of both WT and AF251705 ${ }^{-1-}$ mice (Supplementary Fig. 2). Although PBSliposome-treated WT mice showed significantly longer survival than PBS-liposome-treated AF251705 ${ }^{-1}$ mice, $\mathrm{Cl}_{2} \mathrm{MBP}-$ liposome-treated WT mice showed increased mortality at the same level as $\mathrm{Cl}_{2} \mathrm{MBP}$-liposome-treated $A F 251705^{-1-}$ mice (Fig. 2c). Taken together, these results suggest that MAIR-II regulates the influx of $\mathrm{M} \phi / \mathrm{Mo}$ into the peritoneal cavity, which contributes to the longer survival of WT mice after CLP.

WT iMo protects $A F 251705^{-/-}$mice from peritonitis. iMo are rapidly recruited to sites of infection and inflammation, and they play a critical protective role in the CLP model ${ }^{5-7,11}$. As we found that MAIR-II is highly expressed on iMo (Ly6G ${ }^{-}$F4/80high Ly6Chigh), but not on both patrolling monocytes (Ly6G ${ }^{-} \mathrm{F} 4 /$ $80^{\text {low }}$ Ly6C low $)$ and neutrophils (Ly6G high F4/80 ${ }^{\text {low }}$ ) (Fig. 2d), we investigated whether MAIR-II expressed on iMo is involved in protecting mice from CLP-induced peritonitis. AF251705 mice were injected with iMo purified from either WT or AF251705 $-1-$ mice, and the mice then underwent CLP. We found that $A F 251705^{-I-}$ mice that received WT iMo showed significantly improved survival after CLP compared with mice that received $A F 251705^{-1-}$ iMo (Fig. 2e). Moreover, although phagocytosis of Escherichia coli was comparable in WT and AF251705 $-1-$ iMo (Supplementary Fig. 3), the numbers of peritoneal aerobic bacteria were reduced in mice that received WT iMo compared with mice that received $A F 251705^{-1-}$ iMo (Fig. 2f). These results indicate that MAIR-II expressed on iMo plays an important role in protecting against septic peritonitis, suggesting that MAIR-II is involved in the migration of iMo into the peritoneal cavity.

To test this hypothesis, carboxyfluorescein diacetate succinimidyl ester (CFSE)-labelled iMo from WT or $A F 251705^{-1-}$ mice were adoptively transferred to $A F 251705^{-/}$mice immediately before CLP. Significantly more CFSE-labelled iMo were detected in the peritoneal cavity of mice that received CFSElabelled WT iMo than in mice that received CFSE-labelled $A F 251705^{-1-}$ iMo at $20 \mathrm{~h}$ after CLP (Fig. 2g), suggesting that AF $251705^{-1-}$ iMo were impaired in migration into the peritoneal cavity compared with WT iMo. To examine whether this is derived from an intrinsic defect of $A F 251705^{-/-}$iMo 

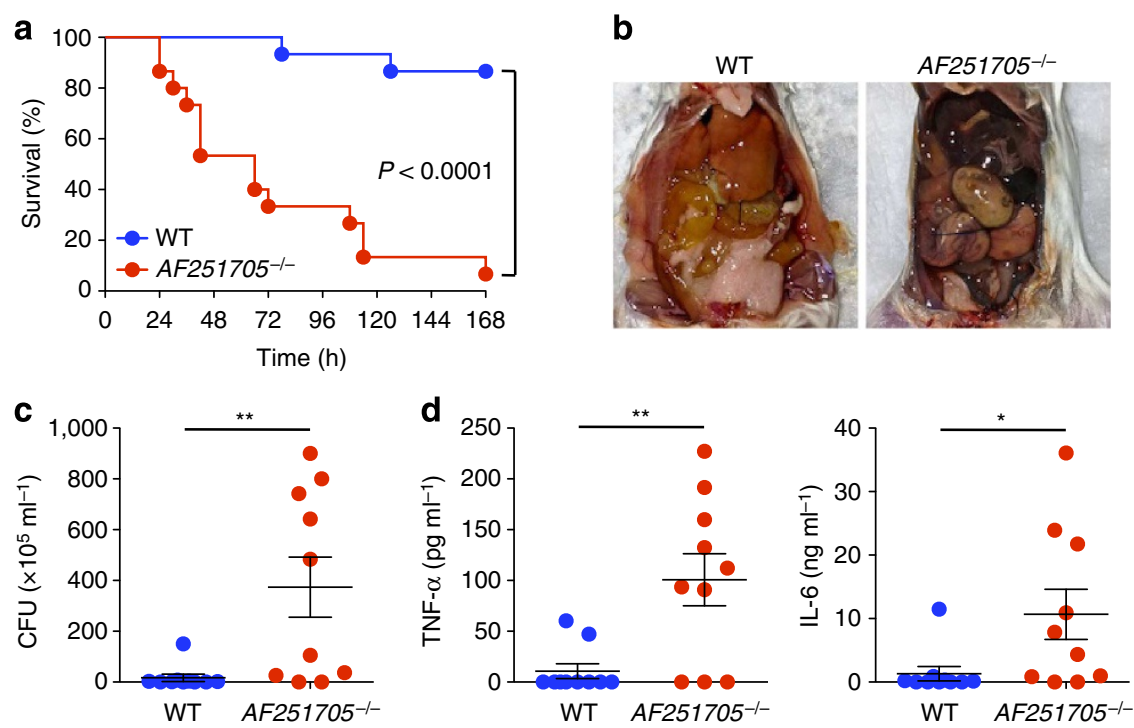

Figure 1 | AF251705 / $^{-}$mice are more susceptible to CLP-induced peritonitis than WT mice. (a) WT and AF251705 $/-$mice ( $n=15$ per group) were subjected to CLP. Mice were monitored every $6 \mathrm{~h}$ for 7 days to determine survival. (b) Representative features of the peritoneal cavity of WT or AF251705 $-/-$ mice $20 \mathrm{~h}$ after CLP. (c) Bacterial numbers in the peritoneal cavity of WT or AF251705-/- mice $(n=10$ per group) $20 \mathrm{~h}$ after CLP. (d) Plasma levels of TNF- $\alpha$ and IL-6 $20 \mathrm{~h}$ after CLP in WT or AF251705-/- mice ( $n=10$ per group). CFU, colony forming unit. ${ }^{\star} P<0.05,{ }^{\star \star} P<0.01$ (log-rank test in $\mathbf{a}$, Mann-Whitney $U$-analyses in $\mathbf{c}$ and two-tailed Student's $t$-test in $\mathbf{d}$ ). Data are representative of three (a) or two (b-d) independent experiments. Error bars indicate s.d. (c,d).

function, 1:1 mixture of CFSE-labelled WT $\left(\mathrm{CD} 45.1^{+}\right)$and $A F 251705^{-1-}\left(\mathrm{CD} 45.2^{+}\right)$iMo were adoptively transferred to AF $251705^{-1-}$ mice immediately before CLP. We observed again that significantly more WT $\left(\mathrm{CD} 45.1^{+}\right)$iMo were detected in the peritoneal cavity than $A F 251705^{-/-}\left(\mathrm{CD} 45.1^{-}\right)$iMo at $20 \mathrm{~h}$ after CLP (Fig. 2h). These results indicate that MAIR-II plays an important role in the migration of iMo to sites of inflammation in the peritoneal cavity after CLP.

VLA-4 is involved in iMo migration. Although CCR2 expressed on iMo and its ligand CC-chemokine ligand 2 (CCL2) play an important role in the egress of iMo from the bone marrow to the peripheral blood ${ }^{12}$, several lines of evidence demonstrate that the interaction between CCR2 and CCL2 is not involved in the migration of iMo from the peripheral blood to sites of infection ${ }^{4}$. Indeed, the concentration of CCL2 in lavage fluid taken from the peritoneal cavity after CLP and the expression of CCR2 on iMo were comparable in WT and $A F 251705^{-1-}$ mice (Supplementary Fig. 4a,b). Further studies did not show any difference in the levels of other chemokines and cytokines in the lavage fluid after CLP (Supplementary Fig. 4c).

Previous reports demonstrated that the recruitment of iMo from the blood to sites of infection requires adhesion molecules such as VLA-4 (refs 13,14). To examine whether VLA-4 is also involved in the recruitment of iMo into the peritoneal cavity after CLP, mice were injected with either a monoclonal antibody $(\mathrm{mAb})$ against VLA-4 or LFA-1 immediately before CLP. The number of $\mathrm{M} \phi / \mathrm{Mo}$ in the peritoneal cavity after CLP in mice that were injected with anti-VLA-4 mAb was significantly lower than in mice that received control antibody. However, the neutrophil number after CLP was comparable between these two groups (Fig. 3a). By contrast, mice that were treated with anti-LFA-1 $\mathrm{mAb}$ showed significantly lower number of neutrophils, but not $\mathrm{M} \phi / \mathrm{Mo}$, in the peritoneal cavity after CLP compared with mice that were treated with control antibody (Fig. 3a). These results suggest that VLA-4 and LFA-1 play an important role in transmigration of iMo and neutrophils, respectively, into the peritoneal cavity after CLP.

To directly demonstrate the involvement of VLA-4 in migration of iMo to the peritoneal cavity after CLP, CFSElabelled iMo from WT or $A F 251705^{-1}$ mice were adoptively transferred to $A F 251705^{-1-}$ mice. These mice were treated with either anti-VLA-4 mAb or control antibody immediately before CLP. Significantly more CFSE-labelled WT iMo were detected in the peritoneal cavity of mice that were treated with control antibody compared with in mice that were treated with antiVLA-4 mAb (Fig. 3b). However, treatment with anti-VLA-4 mAb did not affect the number of CFSE-labelled AF251705-I- iMo (Fig. 3b). Taken together, these results indicate that VLA-4 as well as MAIR-II plays a critical role in transmigration of iMo into the peritoneal cavity after CLP.

TLR4 and MyD88 are involved in iMo migration. We previously reported that simultaneous stimulation of peritoneal macrophages with an anti-MAIR-II $\mathrm{mAb}$ and LPS synergistically increased the production of proinflammatory cytokines such as TNF- $\alpha^{17}$, suggesting that MAIR-II functionally associates with TLR4. Recent evidence also suggests that MyD88 and TLR4 are required for protection from CLP-induced peritonitis ${ }^{25,26}$. To examine whether iMo require the interaction of MAIR-II with TLR4 signalling for their recruitment after CLP, we adoptively

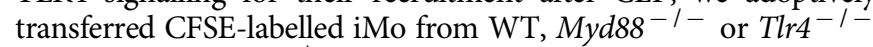
mice into $A F 251705^{-I-}$ mice, as well as WT mice. Recruitment of iMo from $M y d 88^{-I-}$ and Tlr4 ${ }^{-I-}$ mice into the peritoneal cavity of both $A F 251705^{-/-}$and WT mice was impaired compared with the recruitment of iMo from WT mice (Fig. 4a and Supplementary Fig. 5). Moreover, the adoptive transfer of iMo from WT, but not $M y d 88^{-1-}$ and $T l r 4^{-7-}$, mice improved survival in $A F 251705^{-1-}$ mice after CLP (Fig. 4b). These results suggest that TLR4/MyD88 signalling is critical for the recruitment of iMo into the peritoneal cavity after CLP.

LPS induces association of MAIR-II with TLR4 and FcR $\gamma$ chain. We next examined whether MAIR-II is physically and 
a

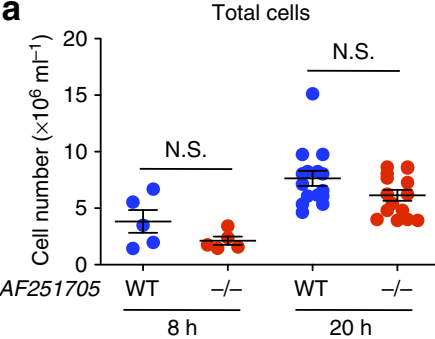

b

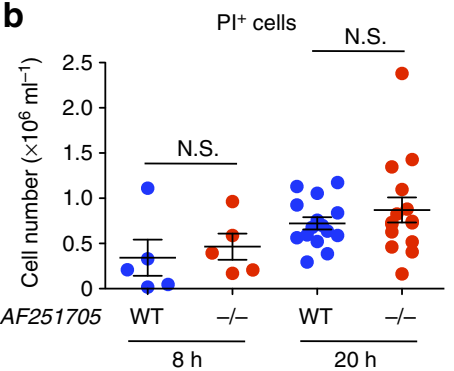

d
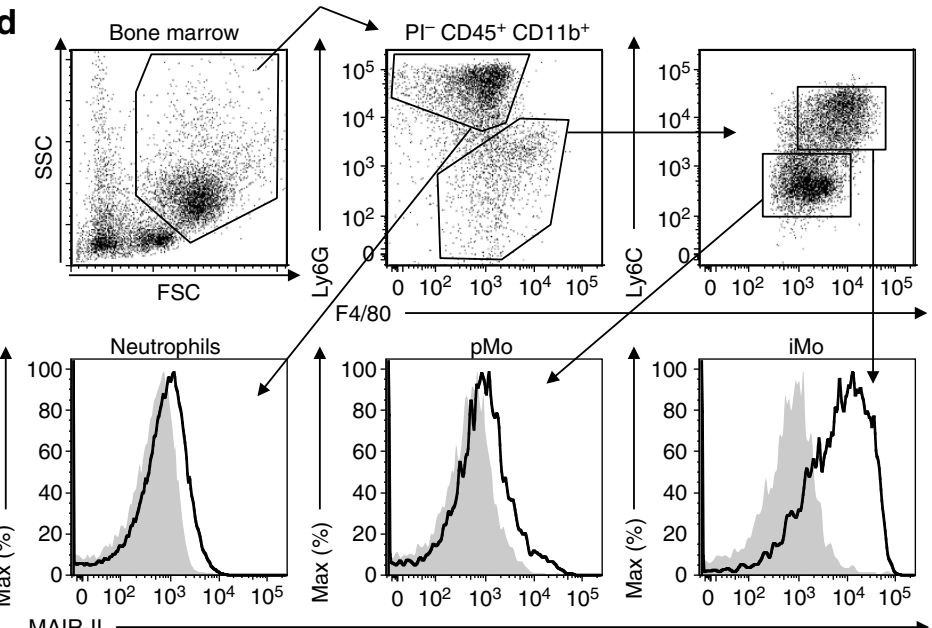

C

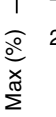

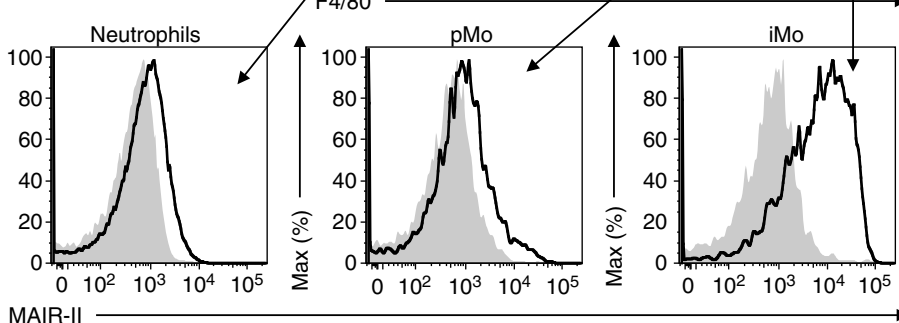

$\mathbf{e}$
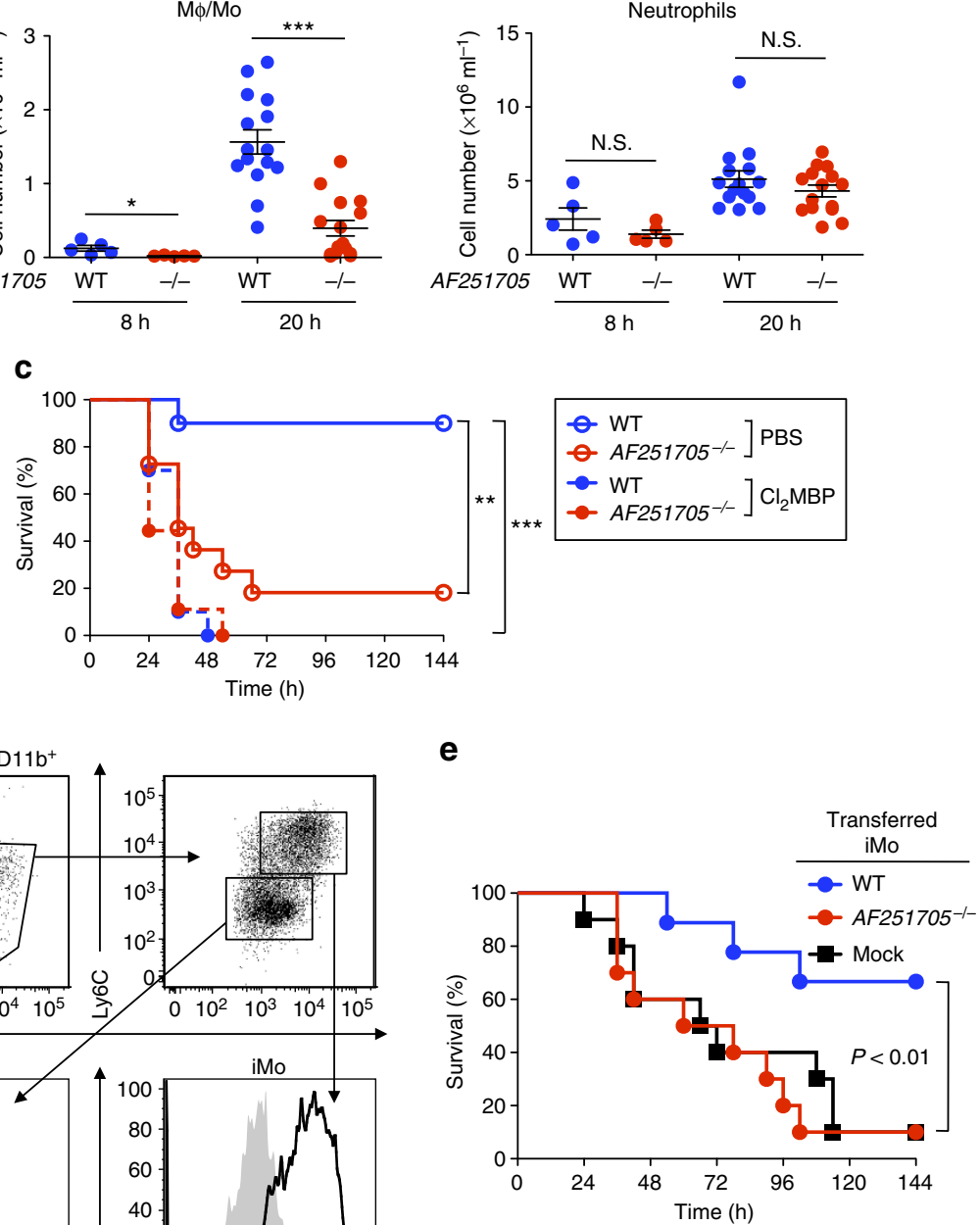

f

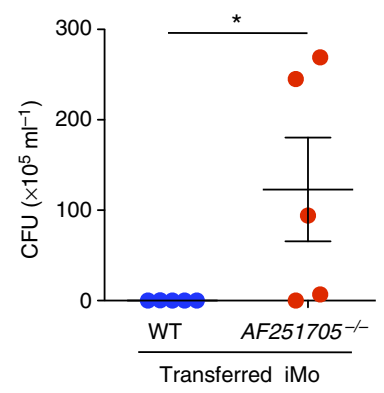

g Transferred iMo

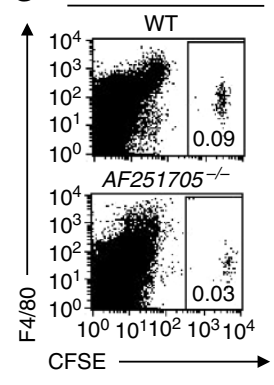

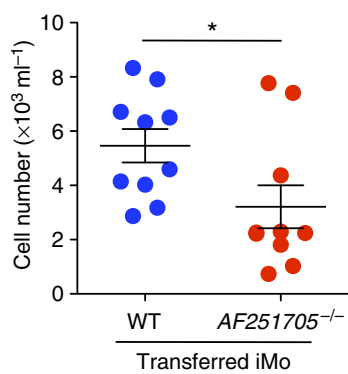

h
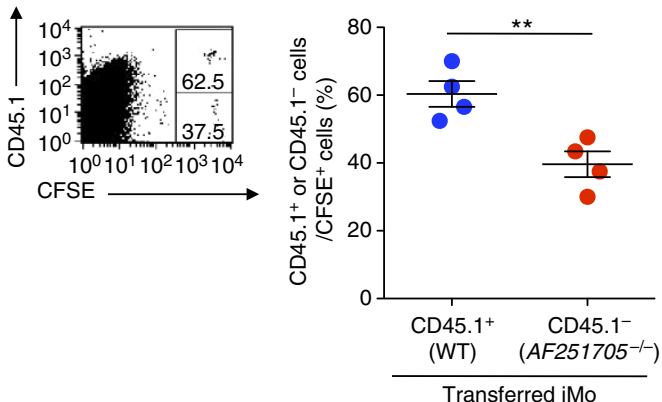

Figure 2 | MAIR-II regulates the influx of iMo to the peritoneal cavity after CLP. (a,b) Numbers of total cells, M申/Mo $\left(\mathrm{CD} 11 \mathrm{~b}^{+} \mathrm{F} 4 / 80^{+}\right)$, neutrophils $\left(\mathrm{CD} 11 \mathrm{~b}{ }^{+} \mathrm{F} 4 / 80^{-} \mathrm{Ly}_{6 \mathrm{G}}{ }^{+}\right)$and apoptotic cells $\left(\mathrm{PI}^{+}\right)$in the peritoneal cavity of WT or AF251705-/- mice, $8(n=5)$ or 20 h $(n=15)$ after CLP operation (c) Liposome-encapsulated PBS- or liposome-encapsulated dichloromethylene bisphosphate $\left(\mathrm{Cl}_{2} \mathrm{MBP}\right)$-treated WT and $A F 251705^{-/-}$mice were subjected to CLP. Mice were monitored every $6 \mathrm{~h}$ for 6 days to determine survival ( $n=9-11$ per group). (d) Flow cytometry analysis of MAIR-II expression on iMo (Ly6G - F4/80 high Ly6Chigh), patrolling monocytes (pMo) (Ly6G- F4/80 low Ly6Clow) and neutrophils (Ly6Ghigh F4/80 low) in the bone marrow. (e,f) iMo from WT or AF251705 ${ }^{-1}$ - mice were intravenously transferred into AF251705 ${ }^{-/}$- mice after CLP and monitored every $6 \mathrm{~h}$ for 6 days to determine survival ( $n=10$ per group). Mock indicates mice that received PBS alone after CLP (e). Bacterial titres in the peritoneal cavity $20 \mathrm{~h}$ after CLP were determined by colony-forming unit (CFU) assay (f). (g) CFSE-labelled iMo from WT or AF251705-/ - mice were intravenously transferred into AF251705-/- mice $20 \mathrm{~h}$ after CLP. Numbers of migrated CFSE ${ }^{+}$iMo in the peritoneal cavity were analysed by using flow cytometry $20 \mathrm{~h}$ after CLP ( $n=10$ per group). (h) The mixture of equal number of CFSE-labelled WT $\left(C D 45.1^{+}\right)$and AF251705 ${ }^{-/-}\left(C D 45.2^{+}\right)$iMo were intravenously transferred into AF251705 ${ }^{-/-}$mice immediately before CLP. The proportions of transferred CD45.1 ${ }^{+}(\mathrm{WT})$ and CD45.1 $\left(A F 251705^{-/-}\right)$iMo in the peritoneal cavity were analysed by using flow cytometry $20 \mathrm{~h}$ after CLP ( $n=4$ per group). N.S., not significant. ${ }^{\star} P<0.05,{ }^{\star \star} P<0.01$ and ${ }^{\star \star \star} P<0.001$ (two-tailed Student's $t$-test in (a,b,g,h), log-rank test in $(\mathbf{c}, \mathbf{e})$ and Mann-Whitney $U$-analyses in $\mathbf{f})$. Error bars indicate s.d. Data are representative of two independent experiments. 

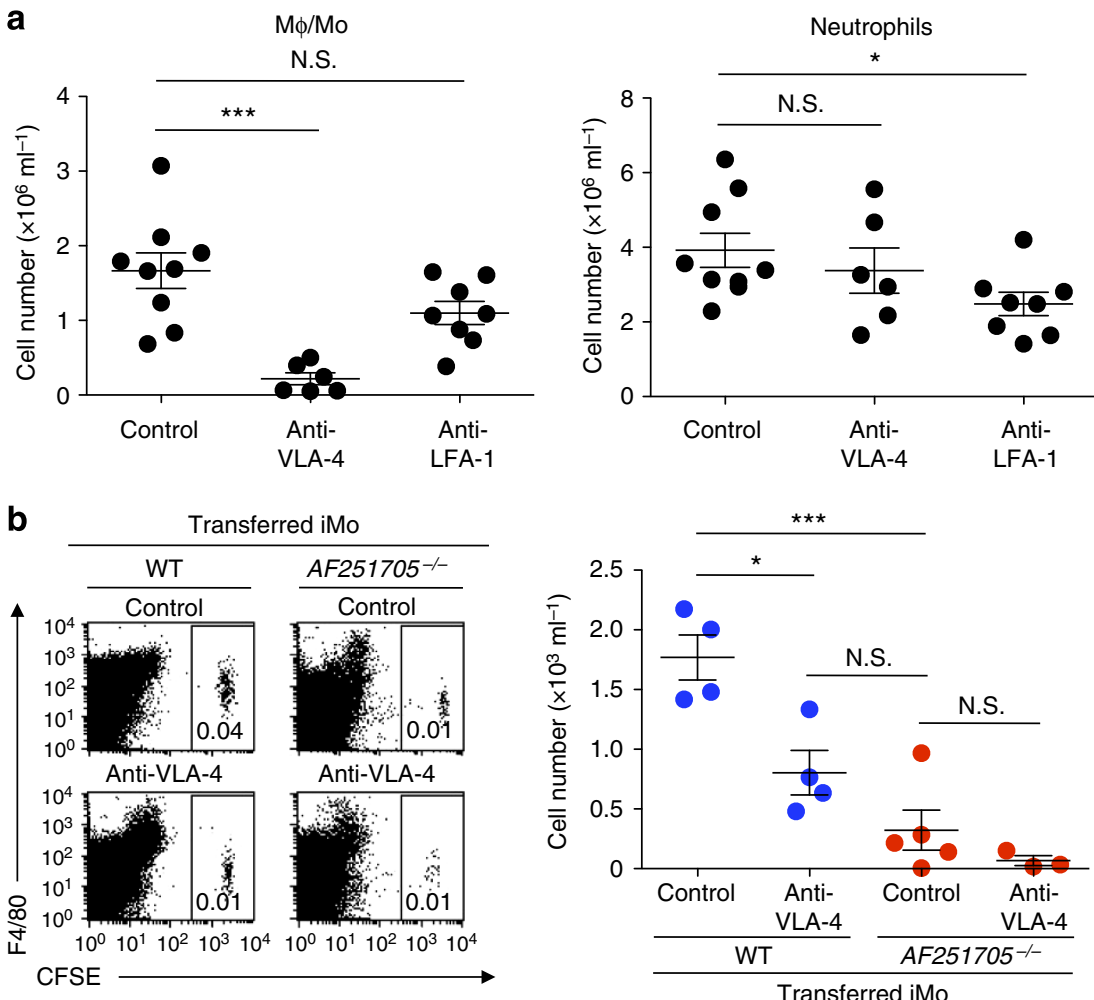

Figure 3 | VLA-4 plays a critical role in transmigration of iMo into the peritoneal cavity after CLP. (a) Mice were treated with control antibody, antiVLA-4 mAb or anti-LFA-1 mAb immediately before CLP. Numbers of M $\phi / M o\left(C D 11 b^{+}\right.$F4/80 ${ }^{+}$) and neutrophils $\left(C D 11 b^{+}\right.$F4/80 ${ }^{-}$Ly6G $\left.{ }^{+}\right)$in the peritoneal cavity $20 \mathrm{~h}$ after CLP operation ( $n=6-9$ per group). (b) CFSE-labelled iMo from WT or AF251705 ${ }^{-/}$mice were adoptively transferred intravenously into AF251705-/ - mice. These mice were treated with anti-VLA-4 mAb or control antibody immediately before CLP. Migrated CFSE ${ }^{+}$iMo $^{-1}$ in the peritoneal cavity were counted by using flow cytometry $20 \mathrm{~h}$ after CLP ( $n=4$ or 5 per group). N.S., not significant. ${ }^{\star} P<0.05$ and ${ }^{\star \star \star} P<0.001$ (twotailed Student's $t$-test in $\mathbf{a}, \mathbf{b}$ ). Error bars indicate s.d. Data are representative of two independent experiments.
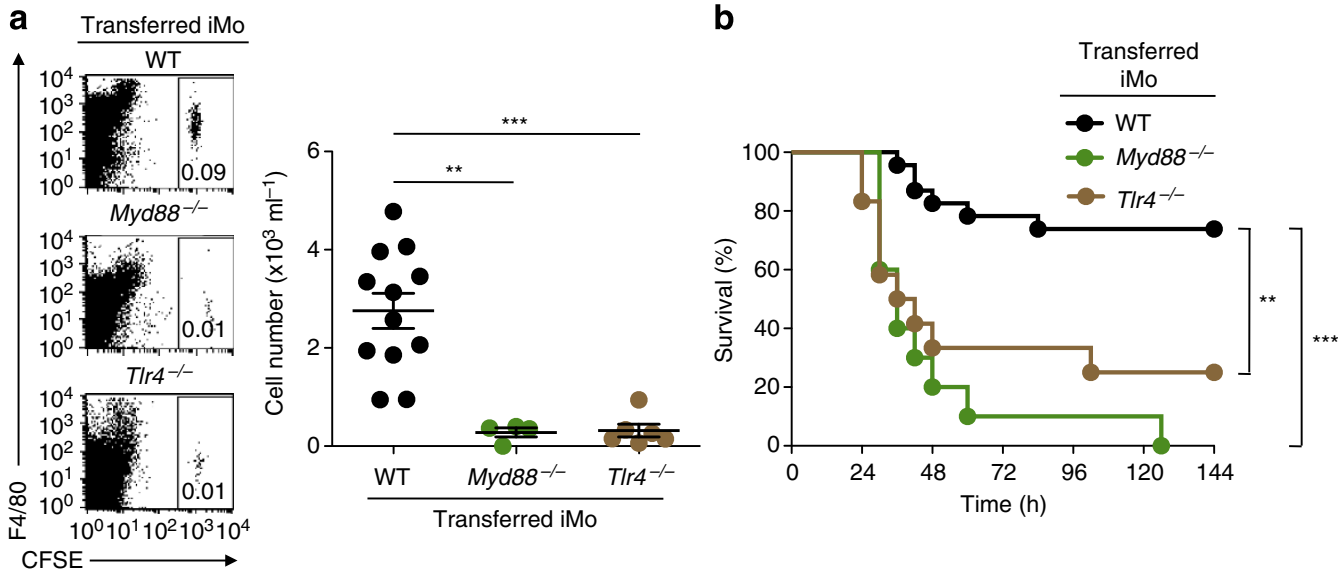

Figure 4 | iMo require TLR4 and MyD88 signalling for their recruitment. (a,b) CFSE-labelled (a) or unlabelled (b) iMo from WT, Myd88 ${ }^{-/}-$or TIr4 $^{-/}$

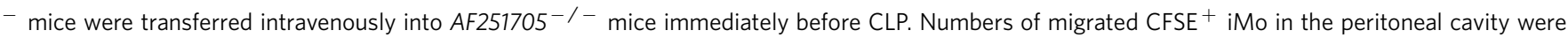
counted $20 \mathrm{~h}$ after $\operatorname{CLP}$ ( $n=4,6$ or 12 per group) (a). Mice were monitored every $6 \mathrm{~h}$ for 6 days to determine survival ( $n=10$ per group) (b). ${ }^{\star \star} P<0.01$ and ${ }^{\star \star \star} P<0.001$ (two-tailed Student's $t$-test in $\mathbf{a}$ and log-rank test in $\mathbf{b}$ ). Error bars indicate s.d. (a). Data are representative of two independent experiments.

functionally associated with TLR4 by using RAW264.7 macrophage transfectants stably expressing Flag-tagged MAIR-II or Flagtagged CD300a, which is highly homologous to MAIR-II in the extracellular domain ${ }^{16}$. Although both MAIR-II and CD300a were not coimmunoprecipitated with TLR4 in the steady state, MAIR-II, but not CD300a, was coimmunoprecipitated with TLR4 after LPS stimulation (Fig. 5a and Supplementary Figs 6a and 7). As both
RAW264.7 transfectants expressing Flag-tagged CD300a and MAIR-II expressed comparable amount of Flag protein (Supplementary Fig. 8), these results indicated that MAIR-II, but not CD300a, specifically associates with TLR4 after stimulation with LPS. To investigate whether FcR $\gamma$ chain is involved in this association, we further analysed the association of TLR4 with FcR $\gamma$-interacting mouse CD300 molecules MAIR-IV/CLM-5/ 
a

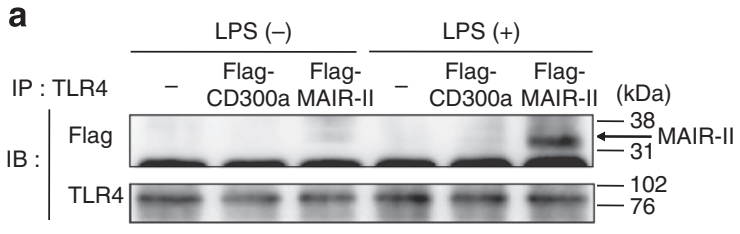

b

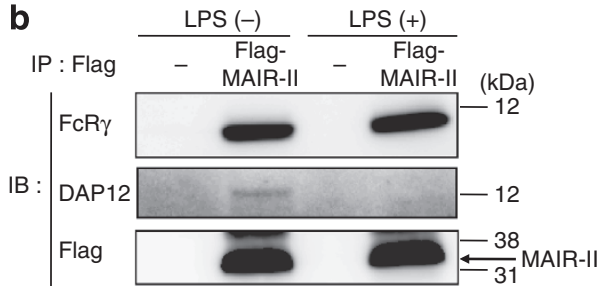

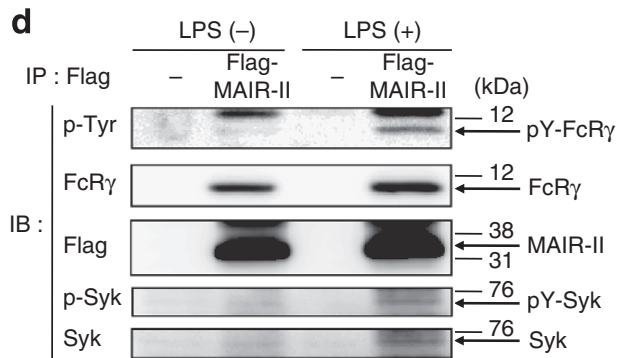
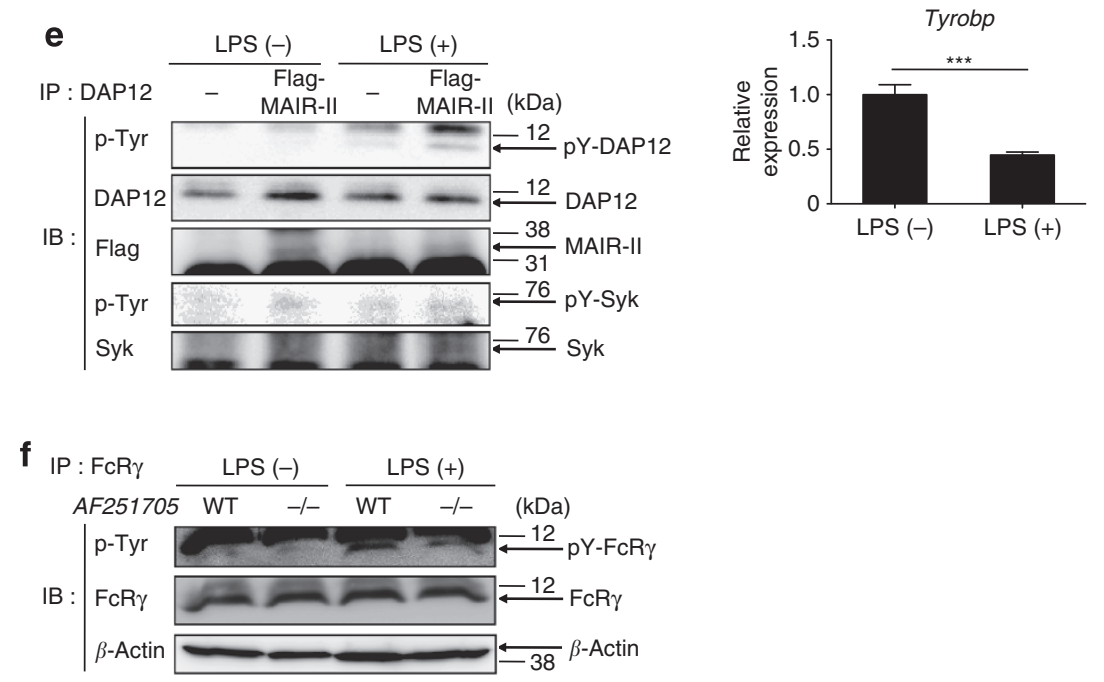

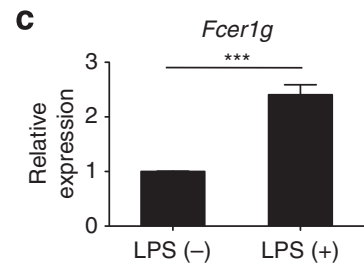

C 
a
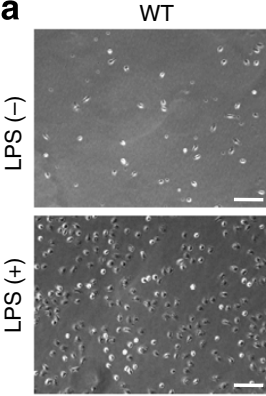

C

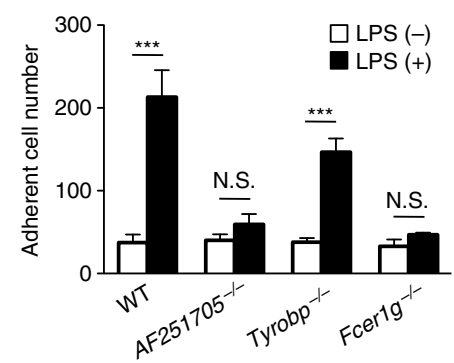

e

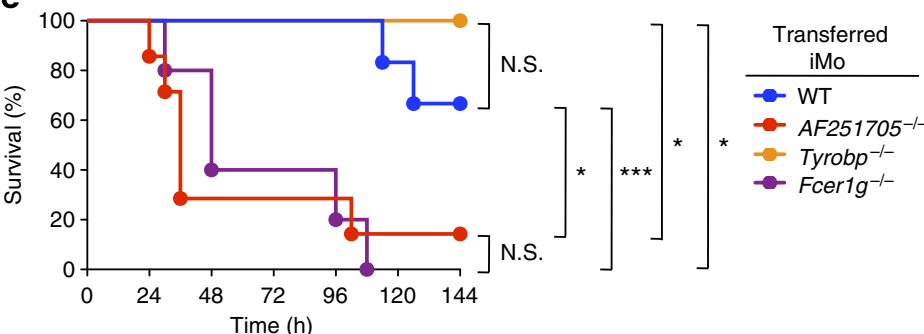

g

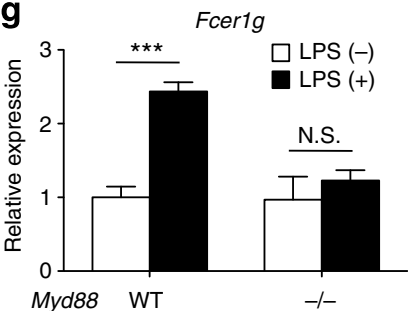

AF $251705^{-/}$
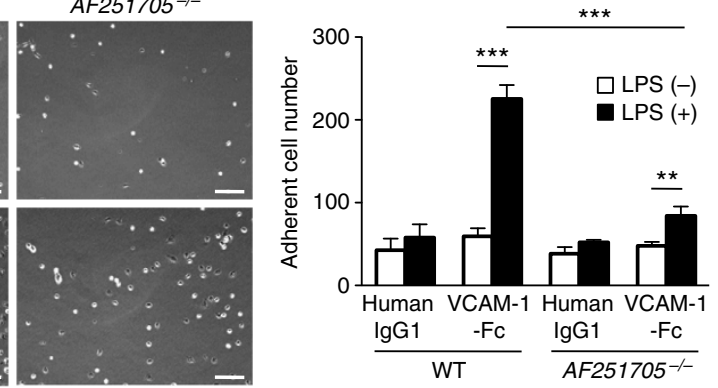

d

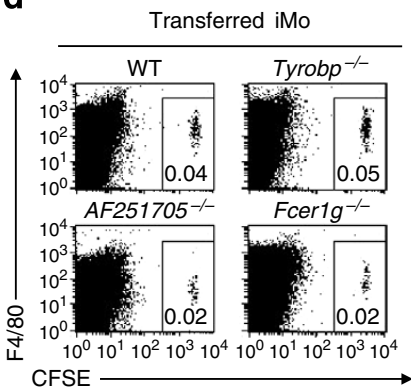

b
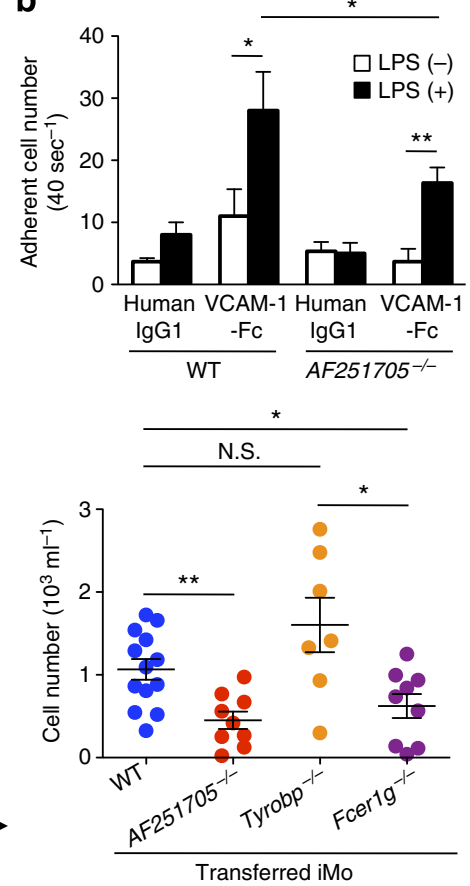

f

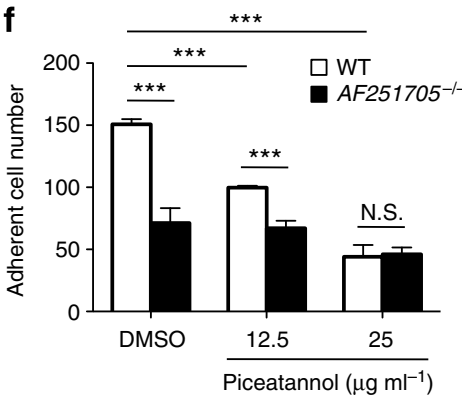

h

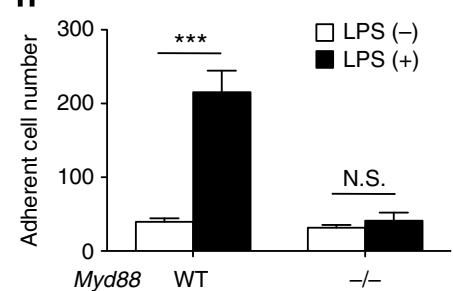

Figure 6 | iMo require FcR $\gamma$ chain and Syk for migration into the peritoneal cavity after CLP. (a,c) iMo from WT, AF251705 ${ }^{-/-}$, Tyrobp ${ }^{-/-}$or Fcer1g ${ }^{-/}$ - mice were incubated on human IgG1-precoated or VCAM-1-Fc-precoated plates in the presence or absence of LPS for $20 \mathrm{~h}$, and adherent cells were counted by means of microscopy ( $n=3$ per group). Scale bars, $100 \mu \mathrm{m}$. (b) iMo from WT or AF251705 ${ }^{-1-}$ mice were incubated in the presence or

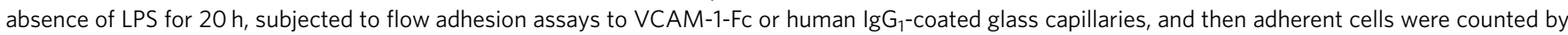
means of microscopy ( $n=3$ per group). (d) CFSE-labelled iMo from WT, AF251705 $-1-$, Tyrobp ${ }^{-/-}$or Fcer1 ${ }^{-/-}$mice were transferred intravenously into AF251705- / mice $20 \mathrm{~h}$ after CLP. Migrated CFSE ${ }^{+}$iMo in the peritoneal cavity were counted by using flow cytometry $20 \mathrm{~h}$ after CLP ( $n=7,9$ or 13 per group). (e) iMo from WT, AF251705 ${ }^{-1}$, Tyrobp ${ }^{-/-}$or Fcer1g ${ }^{-/}$mice were transferred intravenously into AF251705 $5^{-/-}$mice immediately before CLP. Mice were monitored every $6 \mathrm{~h}$ for 6 days to determine survival $\left(n=6,7,3\right.$ or 5 per group). (f) iMo from WT or AF251705 ${ }^{-/-}$mice were stimulated with LPS on VCAM-1-Fc-precoated plates in the presence or absence of piceatannol, a Syk inhibitor, for $20 \mathrm{~h}$, and adherent cells were counted by means of microscopy ( $n=3$ per group). (g) iMo from WT or Myd88-/- mice were left unstimulated or stimulated with LPS for $20 \mathrm{~h}$, and the expression of Fcer1g and Tyrobp mRNA was analysed by using real-time reverse transcription-PCR ( $n=3$ per group). (h) iMo from WT or Myd88 ${ }^{-/-}$mice were incubated on VCAM-1-Fc-precoated plates in the presence or absence of LPS for $20 \mathrm{~h}$, and adherent cells were counted by means of microscopy ( $n=3$ per group). ${ }^{\star} P<0.05,{ }^{\star \star} P<0.01$ and ${ }^{\star \star \star} P P<0.001$ (two-tailed Student's $t$-test in (a-d,f-h)) and log-rank test in $\left.\mathbf{e}\right)$. N.S., not significant. Error bars indicate s.d. Data are representative of two independent experiments. See also Supplementary Figs 9 and 10.

than that of $A F 251705^{-1-}$ iMo. Similarly, LPS stimulation did not enhance the adhesion of Fcer $1 g^{-1-}$ iMo to VCAM-1-Fc (Fig. 6c and Supplementary Fig. 9c). In contrast, Tyrobp ${ }^{-1-}$ iMo showed enhanced adhesion after LPS stimulation (Fig. $6 c$ and
Supplementary Fig. 9c). Moreover, iMo from Fcer $1 g^{-1-}$ or AF $251705^{-1-}$ mice showed impaired migration into the peritoneal cavity compared with those from WT mice (Fig. 6d). In contrast, migration of Tyrobp $p^{-I-}$ iMo into the peritoneal 
cavity was comparable with WT iMo (Fig. 6d). In agreement with these results, the adoptive transfer of iMo from WT and Tyrobp ${ }^{-1-}$, but not $A F 251705^{-1-}$ and Fcerlg ${ }^{-1-}$, mice improved survival of $A F 251705^{-1-}$ mice after CLP (Fig. 6e). Together, these results indicate that FcR $\gamma$ chain, but not DAP12, plays an important role in the TLR4/MAIR-II-mediated adhesion of iMo to VCAM-1, in accordance with the results that MAIR-II associated with FcR $\gamma$ chain, but not DAP12 after LPS stimulation.

To examine whether FcR $\gamma$ chain-mediated Syk activation is required for iMo adhesion to VCAM-1, WT or AF251705 - IiMo were incubated on culture dishes precoated with VCAM-1Fc in the presence or absence of piceatannol, a Syk inhibitor. Piceatannol suppressed iMo adhesion to VCAM-1-Fc in a dosedependent manner (Fig. 6f and Supplementary Fig. 10a,b), suggesting that Syk activation is required for LPS-induced iMo adhesion to VCAM-1.

E-selectin-mediated integrin activation was regulated via the activation of Syk, subsequent Bruton's tyrosine kinase (Btk), phosphoinositide 3-kinase and phospholipase C (PLC) $\gamma_{2}$ (ref. 33). We examined whether these molecules in the downstream of Syk were also involved in the adhesion of iMo to VCAM-1-Fc by using an inhibitor of the activity for each molecule. Incubation of WT iMo in the presence of PCI-32765 (a Btk inhibitor), Wortmannin and LY294002 (both phosphoinositide 3kinase inhibitors), or U-73122 (a PLC inhibitor) showed significantly impaired adhesion to VCAM-1-Fc, which was comparable with $A F 251705^{-1-}$ iMo (Supplementary Fig. 10c). These results suggest that MAIR-II expressed on iMo regulates TLR4-mediated cell adhesion to VCAM-1 via the activation of Syk.

MyD88 regulates FcR $\gamma$ and DAP12 expression and iMo adhesion. We have shown that the migration of iMo from Tlr $4^{-1-}$ and $M y d 88^{-1-}$ mice into the peritoneal cavity was impaired compared with WT iMo, and that these mice showed significantly shorter survival than WT mice after CLP (Fig. 4). Furthermore, LPS stimulation upregulated $\mathrm{FcR} \gamma$ chain expression and downregulated DAP12 expression and adhesion of iMo to VCAM-1. To directly show that LPS-mediated signalling via MyD88 is involved in the regulation of the expression of both $\mathrm{FcR} \gamma$ chain and DAP12, and the adhesion of iMo to VCAM-1, iMo derived from WT or $M y d 88^{-1-}$ mice were stimulated with LPS. In accordance with the results shown in Fig. 5c, LPS stimulation increased Fcerlg expression but decreased Tyrobp expression in WT iMo (Fig. 6g). However, the expression of Fcer $1 g$ and Tyrobp was not altered in iMo from $M y d 88^{-1-}$ mice (Fig. 6g). Moreover, although LPS stimulation enhanced binding of WT iMo to VCAM-1-Fc, it did not enhance the binding of Myd88-I- iMo (Fig. 6h and Supplementary Fig. 10d). These results indicate that MyD88 is required for the regulation of $\mathrm{FcR} \gamma$ chain and DAP12 expression, and the adhesion of iMo to VCAM-1.

\section{Discussion}

In the present study, we first showed that TLR4/MyD88 signalling is essential for iMo recruitment from the blood to sites infected with Gram-negative bacteria via VLA-4. We further showed that TLR4/MyD88-mediated signalling upregulated FcR $\gamma$ chain expression, and induced physical and functional association of TLR4/MyD88 with MAIR-II, FcR $\gamma$ chain and Syk, leading to activation of VLA-4 in iMo (Fig. 7).

Previous studies have demonstrated that TLR4 is coimmunoprecipitated with CD16 (Fc $\gamma$ receptor III), an FcR $\gamma$ chain-coupled immunoreceptor, in neutrophils and macrophages after stimulation with LPS or IgG immune complex ${ }^{34}$. Interestingly, neutrophils and macrophages from $\mathrm{Tlr}^{-1-}$ mice were

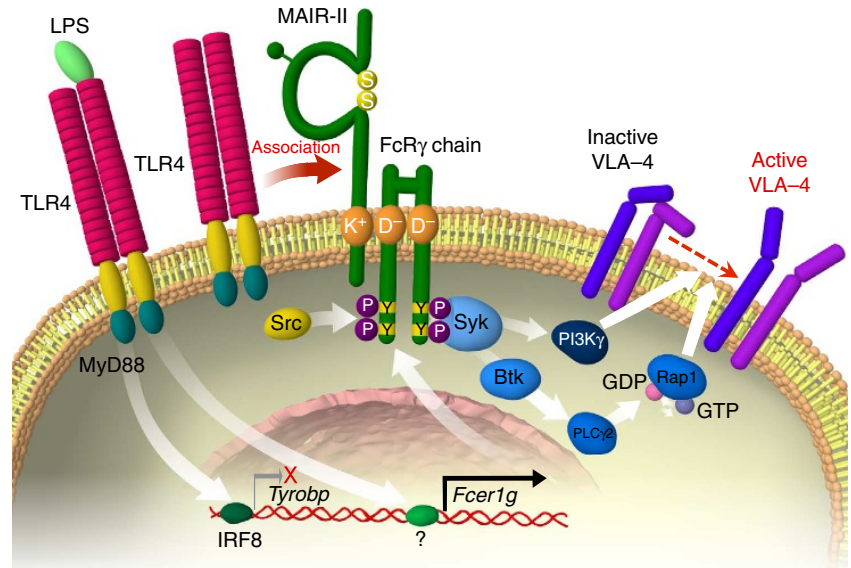

Figure 7 | A proposed model of TLR4-mediated regulation of iMo migration. TLR4/MyD88-mediated signalling upregulated FcR $\gamma$ chain expression but downregulated DAP12 expression, and induced physical and functional association of TLR4/MyD88 with MAIR-II, FcR $\gamma$ chain, and Syk, leading to activation of VLA-4 in iMo. See also text.

unresponsive to either LPS or IgG immune complex for cytokine production and the tyrosine phosphorylation of $\mathrm{FcR} \gamma$ chain on stimulation of CD16 with IgG immune complex ${ }^{34}$, suggesting that TLR4 is indispensable for CD16 signalling via FcR $\gamma$ chain activation. Although it remains undetermined how TLR4 associates with CD16 and MAIR-II, FcR $\gamma$ chain may play a key role in the link between TLR4 and MAIR-II or CD16. As LPS stimulation induces activation of the Src family of kinases, a family of non-receptor tyrosine kinases ${ }^{35}$, it is possible that activated Src kinases phosphorylate the tyrosine of ITAM in FcR $\gamma$ chain. Interestingly, we showed that another $\mathrm{FcR} \gamma$ chaininteracting mouse CD300 molecule CLM-5/MAIR-IV/LMIR4 also associated with TLR4 after stimulation with LPS. In contrast, we did not observe the association of TLR4 with CD300f that also interacts with FcR $\gamma$ chain (Supplementary Fig. 7). It is unclear at present whether $\mathrm{FcR} \gamma$ chain is involved in this association. Further studies are required to clarify the molecular basis of the association of TLR4 with FcR $\gamma$ chain-coupled immunoreceptors.

Immunoreceptors containing positively charged amino acids in the transmembrane regions, present on lymphocytes and myeloid cells, are able to non-covalently associate with any of the ITAMbearing signalling adaptors DAP12, FcR $\gamma$ chain, DAP10 or CD3ל; exceptionally, the NK receptor NKG2D can bind to both DAP12 and DAP10 in T cells ${ }^{36}$. MAIR-II is a unique immunoreceptor that binds either DAP12 or FcR $\gamma$ chain (expressed on peritoneal macrophages, but not macrophages or B cells in the spleen) via a lysine residue located in the transmembrane region of MAIR-II ${ }^{17}$. We have shown that LPS stimulation upregulates $\mathrm{FcR} \gamma$ chain expression $^{17}$, leading to the preferential association of MAIR-II with $\mathrm{FcR} \gamma$ chain. A previous report demonstrated that LPS stimulation of macrophages activates interferon regulatory factor8 (ref. 37), which downmodulates Tyrobp transcription and DAP12 expression ${ }^{38}$. This is one possible explanation for why DAP12 expression is downregulated in iMo by stimulation with LPS. In contrast, the expression of $\mathrm{FcR} \gamma$ chain is regulated by multiple transcription factors, including Sp1, GABP and Elf-1 (ref. 39). However, it is unclear at present whether TLR4 signalling is involved in the transcription mechanism of Fcerlg.

We have shown that MAIR-II regulates TLR4-mediated cell adhesion to VCAM-1 in highly purified iMo (in the present study) or cytokine production in $\mathrm{B}$ cells (in a previous report ${ }^{18}$ ) in response to TLR ligands alone, suggesting that a ligand for MAIR-II may be expressed on iMo and B cells, or induced after 
TLR ligand stimulation, resulting in cis-binding between MAIR-II and the ligand. Recent studies suggest that CD300 and the TREM family proteins physically bind to phospholipids ${ }^{40}$. These molecules are preferentially expressed on myeloid cells, whose membrane phospholipids are dynamically remodelled on stimulation with innate stimuli such as TLR ligands ${ }^{41}$, suggesting functional bindings between CD300 and the TREM family proteins. Indeed, ourselves and others have recently identified phosphatidylserine on the outer leaflet of apoptotic cells as a ligand for mouse and human $\mathrm{CD} 300 \mathrm{a}^{42,43}$. Moreover, CD300f is reported to also bind to several extracellular lipids, including phosphatidylserine, ceramide and lipoproteins ${ }^{44-46}$. Therefore, it is possible that MAIR-II may also bind to phospholipids as functional ligands. Our previous study demonstrated that unlike CD300a, MAIR-II does not bind phosphatidylserine ${ }^{47}$. Further studies are required for the clarification of MAIR-II function in vivo.

\section{Methods}

Mice. BALB/c and C57BL/6J mice were purchased from Clea Japan. AF251705 ${ }^{-1-}$ mice were generated in our laboratory and backcrossed onto the C57BL/6J genetic background for 12 generations ${ }^{18}$. C57BL/6.SJL $\left(\right.$ Ptprc $\left.^{a} P e p c^{b}, \mathrm{CD}^{2} 5.1^{+}\right)$mice were purchased from The Jackson Laboratory. $M y d 88^{-/-}$and Tlr $4^{-/-}$mice in a BALB/ c background were purchased from Oriental Bioservice. Tyrobp ${ }^{-1-}$ and Fcerlg ${ }^{-1-}$ mice were provided by Toshiyuki Takai (Tohoku University, Japan) and backcrossed onto the C57BL/6J genetic background for 12 generations as described ${ }^{17,48,49}$. All mice used were 8-12 weeks old female or male. All experiments were performed in accordance with the guidelines of the animal ethics committee of the University of Tsukuba Animal Research Center.

Caecal ligation and puncture. CLP was performed as described ${ }^{42,50}$. The caecum was exposed by a 1 - to $2-\mathrm{cm}$ midline incision in the ventral abdomen, ligated at $\sim 7 \mathrm{~mm}$ from its distal portion and punctured twice with a 25-gauge needle in the ligated segment. The abdomen was closed in two layers, and $1 \mathrm{ml}$ of sterile saline was administered subcutaneously. Sham-operated mice were subjected to a similar laparotomy without ligation and puncture. Peritoneal lavage fluid and plasma from mice 8 and $20 \mathrm{~h}$ after CLP, respectively, were collected for analyses of cytokine and chemokine levels. To determine bacterial loads after CLP, peritoneal lavage fluid from mice $20 \mathrm{~h}$ after CLP was placed on ice and serially diluted in sterile PBS. A $100-\mu \mathrm{l}$ aliquot of each dilution was spread on Brain Heart Infusion agar plates without antibiotics and incubated under aerobic conditions at $37^{\circ} \mathrm{C}$ for $24 \mathrm{~h}$.

Measurement of cytokines and chemokines. Cytokine and chemokine concentrations were measured in triplicate by using ELISA kits purchased from BD Biosciences (TNF- $\alpha$ and IL-6) and R\&D Systems (CCL2).

Antibodies and flow cytometric analysis. mAbs against mouse CD11b, Ly6G, Ly6C, CD45.1, CD45.2 and LFA-1 (CD11a) (M1/70, 1A8, AL-21, A20, 104 and $\mathrm{M} 17 / 4$, respectively) were purchased from BD Biosciences. mAbs against mouse F4/ 80 (Cl:A3-1), CCR2 (475301) and VLA-4 (PS/2) were purchased from AbD Serotec, R\&D Systems and Abcam, respectively. mAbs against mouse MAIR-II (TX52) were generated in our laboratory as described ${ }^{16,17}$. mAbs diluted at $10 \sim 50$ times with PBS were used for flow cytometric analysis by using a FACSCalibur or an LSR Fortessa flow cytometer (BD Boscience). For in vivo analyses, $100 \mu \mathrm{g}$ of anti-LFA-1 or anti-VLA-4 were injected intravenously into mice. Cell Quest software (BD Biosciences) and FlowJo software (Tree Star) was used for data analysis.

Depletion of macrophages and monocytes. For in vivo depletion of $\mathrm{M \phi} / \mathrm{Mo}$, mice were injected intravenously with $200 \mu \mathrm{l}$ PBS-liposomes or $\mathrm{Cl}_{2} \mathrm{MBP}$-liposomes (Encapsula NanoSciences) 1 day before CLP, as described ${ }^{51}$.

Isolation of iMo and adoptive transfer. iMo were purified from bone marrow cells by positive selection using a MACS cell separation system (Miltenyi Biotec) with allophycocyanin-conjugated anti-CCR2 $\mathrm{mAb}$ and anti-allophycocyanin MicroBeads (Miltenyi Biotec). The purity of iMo, which were determined as $\mathrm{CD}_{11 \mathrm{~b}}{ }^{+} \mathrm{Ly}_{6 \mathrm{C}} \mathrm{high}^{\mathrm{ig}}$ cells, was $>90 \%$. For adoptive transfer, iMo were transferred by intravenous inoculation of $1 \times 10^{6}$ cells into the orbital venous plexus of AF251705 ${ }^{-I-}$ mice immediately before CLP. To trace the transferred iMo, the cells were labelled with CFSE (Molecular Probes) before adoptive transfer.

Biochemical analysis. RAW264.7 transfectants stably expressing Flag-tagged CD300a or Flag-tagged MAIR-II were generated as described ${ }^{16}$, and incubated in RPMI-1640 (Sigma-Aldrich) containing $10 \%$ fetal bovine serum with or without
LPS (O55:B5; Sigma-Aldrich) $\left(1 \mu \mathrm{g} \mathrm{ml}^{-1}\right)$ for $20 \mathrm{~h}$ at $37^{\circ} \mathrm{C}$ in an atmosphere of $5 \%$ $\mathrm{CO}_{2}$. Cells were lysed in lysis buffer containing $1 \%$ digitonin (Calbiochem) supplemented with protease inhibitors as described ${ }^{17}$. Cell lysates were immunoprecipitated with anti-TLR4 polyclonal antibodies (L-14; Santa Cruz Biotechnology), anti-Flag polyclonal antibodies (F7425; Sigma-Aldrich), antiDAP12 polyclonal antibodies (FL-113; Santa Cruz Biotechnology) or anti-FcR $\gamma$ chain polyclonal antibodies (06-727; Millipore). Immunoprecipitates were resolved by SDS-PAGE, transferred onto polyvinylidene difluoride membranes by electroblotting, immunoblotted with polyclonal antibodies against Flag, TLR4, DAP12, FcR $\gamma$ chain (T2040; US Biological), tyrosine phosphorylated Syk (Tyr525/ 526) (2710; Cell Signaling) or Syk (2712; Cell Signaling), followed by horseradish peroxidase (HRP)-conjugated anti-goat IgG (sc-2768; Santa Cruz Biotechnology) or HRP-conjugated TrueBlot anti-rabbit IgG (18-8816-33; eBioscience). In some cases, HRP-conjugated anti-phosphorylated tyrosine mAbs (4G10; Millipore) was used for immunoblotting.

Complementary DNA synthesis and real-time reverse transcription-PCR. Total RNA was extracted with Isogen reagent (Nippon Gene), and cDNA was synthesized by using a High Capacity RNA-to-cDNA Kit (Applied Biosystems). Real-time reverse transcription-PCR was performed with gene-specific primers (Supplementaly Table 2) and SYBR Green master mix (Applied Biosystems). Expression of each target gene was normalized to that of $\beta$-actin.

Cell adhesion analysis. For static adhesion assay, culture dishes (NUNC, $35 \times 10 \mathrm{~mm}$ ) were precoated with human IgG1 antibody (AG502; Millipore) or mouse VCAM-1-Fc (643-VM; R\&D Systems) $\left(2 \mu \mathrm{g} \mathrm{ml}^{-1}\right)$ for $16 \mathrm{~h}$, washed with PBS and blocked with PBS containing $1 \%$ BSA for $1 \mathrm{~h}$ at room temperature. iMo $\left(4 \times 10^{5}\right.$ per dish) were plated on the culture dishes and incubated in RPMI-1640 containing $10 \%$ fetal bovine serum in the presence or absence of LPS $\left(1 \mu \mathrm{g} \mathrm{ml}^{-1}\right)$ for $20 \mathrm{~h}$ at $37^{\circ} \mathrm{C}$ in an atmosphere of $5 \% \mathrm{CO}_{2}$. Non-adherent cells were then gently removed by washing with PBS, the numbers of adherent cells in five fields were counted under BZ-9000 All-in One Fluorescence Microscope (Keyence) $(\times 20)$ and the mean number of adherent cells were calculated. For analysis of the involvement of kinases in iMo adhesion to VCAM-1, inhibitors of Syk (Piceatannol; Sigma-Aldrich), Btk (PCI-32765; Selleck Chemicals), PI3 kinase (Wortmannin and LY294002; both from Wako Pure Chemical Industries) or PLC (U73122; Calbiochem) were added into the culture media before incubation and iMo adhesion was assessed as described above.

Flow adhesion assay was performed as described previously ${ }^{32}$. Briefly, the inner surface of 0.69-mm-diameter glass capillaries (Drummond) was coated with VCAM-1-Fc or human $\operatorname{IgG}_{1}\left(20 \mu \mathrm{g} \mathrm{ml}^{-1}\right.$ each) at $4{ }^{\circ} \mathrm{C}$ overnight and blocked with fetal bovine serum at room temperature for $5 \mathrm{~min}$. iMo $\left(0.8 \times 10^{6}\right.$ cells per $\left.\mathrm{ml}\right)$ resuspended in RPMI1640 containing 10\% fetal bovine serum were infused into the capillaries at a shear stress of 0.20 dyne $^{-1} \mathrm{~m}^{-2}$ at $37^{\circ} \mathrm{C}$. The rate of flow was controlled by a Harvard PHD 2000 syringe pump (Harvard Apparatus). After 4min stabilization period, the cells were monitored under an inverted light microscope (Olympus IX70) and the number of adhered cells in a $0.60-\mathrm{mm}^{2}$ microscope field was determined at a 40 -s interval.

Statistical analysis. Statistical significance between groups was determined by using the two-tailed Student's $t$-test (GraphPad Prism). Bacterial counts of mice subjected to CLP were analysed by using the Mann-Whitney $U$-analysis (GraphPad Prism). Mortality of mice subjected to CLP was analysed by using KaplanMeir survival curves and the log-rank test (GraphPad Prism). Differences were considered significant at $P<0.05$.

\section{References}

1. Gordon, S. \& Taylor, P. R. Monocyte and macrophage heterogeneity. Nat. Rev Immunol. 5, 953-964 (2005).

2. Geissmann, F., Jung, S. \& Littman, D. R. Blood monocytes consist of two principal subsets with distinct migratory properties. Immunity 19, 71-82 (2003)

3. Daley-Bauer, L. P., Wynn, G. M. \& Mocarski, E. S. Cytomegalovirus impairs antiviral CD $8+\mathrm{T}$ cell immunity by recruiting inflammatory monocytes. Immunity 37, 122-133 (2012).

4. Shi, C. \& Pamer, E. G. Monocyte recruitment during infection and inflammation. Nat. Rev. Immunol. 11, 762-774 (2011).

5. Kim, Y. G. et al. The Nod2 sensor promotes intestinal pathogen eradication via the chemokine CCL2-dependent recruitment of inflammatory monocytes. Immunity 34, 769-780 (2011).

6. Dunay, I. R. et al. Gr1 (+) inflammatory monocytes are required for mucosal resistance to the pathogen Toxoplasma gondii. Immunity 29, 306-317 (2008).

7. Iijima, N., Mattei, L. M. \& Iwasaki, A. Recruited inflammatory monocytes stimulate antiviral Th1 immunity in infected tissue. Proc. Natl Acad. Sci. USA 108, 284-289 (2011).

8. Swirski, F. K. et al. Identification of splenic reservoir monocytes and their deployment to inflammatory sites. Science 325, 612-616 (2009). 
9. Swirski, F. K. et al. Ly-6Chi monocytes dominate hypercholesterolemiaassociated monocytosis and give rise to macrophages in atheromata. J. Clin. Invest. 117, 195-205 (2007).

10. Movahedi, K. et al. Different tumor microenvironments contain functionally distinct subsets of macrophages derived from Ly6C(high) monocytes. Cancer Res. 70, 5728-5739 (2010).

11. Ocuin, L. M. et al. Neutrophil IL-10 suppresses peritoneal inflammatory monocytes during polymicrobial sepsis. J. Leukoc. Biol. 89, 423-432 (2011)

12. Serbina, N. V. \& Pamer, E. G. Monocyte emigration from bone marrow during bacterial infection requires signals mediated by chemokine receptor CCR2. Nat. Immunol. 7, 311-317 (2006).

13. Chan, J. R., Hyduk, S. J. \& Cybulsky, M. I. Chemoattractants induce a rapid and transient upregulation of monocyte alpha4 integrin affinity for vascular cell adhesion molecule 1 which mediates arrest: an early step in the process of emigration. J. Exp. Med. 193, 1149-1158 (2001).

14. Henderson, R. B., Hobbs, J. A., Mathies, M. \& Hogg, N. Rapid recruitment of inflammatory monocytes is independent of neutrophil migration. Blood 102, 328-335 (2003).

15. Ley, K., Laudanna, C., Cybulsky, M. I. \& Nourshargh, S. Getting to the site of inflammation: the leukocyte adhesion cascade updated. Nat. Rev. Immunol. 7, 678-689 (2007).

16. Yotsumoto, K. et al. Paired activating and inhibitory immunoglobulin-like receptors, MAIR-I and MAIR-II, regulate mast cell and macrophage activation. J. Exp. Med. 198, 223-233 (2003).

17. Nakahashi, C. et al. Dual assemblies of an activating immune receptor, MAIRII, with ITAM-bearing adapters DAP12 and FcRgamma chain on peritoneal macrophages. J. Immunol. 178, 765-770 (2007).

18. Nakano-Yokomizo, T. et al. The immunoreceptor adapter protein DAP12 suppresses B lymphocyte-driven adaptive immune responses. J. Exp. Med. 208, 1661-1671 (2011).

19. Kumagai, H. et al. Identification and characterization of a new pair of immunoglobulin-like receptors LMIR1 and 2 derived from murine bone marrowderived mast cells. Biochem. Biophys. Res. Commun. 307, 719-729 (2003).

20. Chung, D. H. et al. CMRF-35-like molecule-1, a novel mouse myeloid receptor, can inhibit osteoclast formation. J. Immunol. 171, 6541-6548 (2003).

21. Nakano, T. et al. Activation of neutrophils by a novel triggering immunoglobulin-like receptor MAIR-IV. Mol. Immunol. 45, 289-294 (2008).

22. Clark, G. J., Cooper, B., Fitzpatrick, S., Green, B. J. \& Hart, D. N. The gene encoding the immunoregulatory signaling molecule CMRF-35A localized to human chromosome 17 in close proximity to other members of the CMRF-35 family. Tissue Antigens 57, 415-423 (2001).

23. Nguyen, Q. T. et al. Blockade of CD137 signaling counteracts polymicrobial sepsis induced by cecal ligation and puncture. Infect. Immun. 77, 3932-3938 (2009).

24. Alves-Filho, J. C. et al. Interleukin-33 attenuates sepsis by enhancing neutrophil influx to the site of infection. Nat. Med. 16, 708-712 (2010).

25. Peck-Palmer, O. M. et al. Deletion of MyD88 markedly attenuates sepsisinduced $\mathrm{T}$ and B lymphocyte apoptosis but worsens survival. J. Leukoc. Biol. 83, 1009-1018 (2008).

26. Supajatura, V. et al. Differential responses of mast cell Toll-like receptors 2 and 4 in allergy and innate immunity. J. Clin. Invest. 109, 1351-1359 (2002).

27. Borrego, F. The CD300 molecules: an emerging family of regulators of the immune system. Blood 121, 1951-1960 (2013).

28. Mocsai, A., Ruland, J. \& Tybulewicz, V. L. The SYK tyrosine kinase: a crucial player in diverse biological functions. Nat. Rev. Immunol. 10, 387-402 (2010).

29. Zarbock, A., Lowell, C. A. \& Ley, K. Spleen tyrosine kinase Syk is necessary for E-selectin-induced alpha(L)beta(2) integrin-mediated rolling on intercellular adhesion molecule-1. Immunity 26, 773-783 (2007).

30. Zarbock, A. et al. PSGL-1 engagement by E-selectin signals through Src kinase Fgr and ITAM adapters DAP12 and FcR gamma to induce slow leukocyte rolling. J. Exp. Med. 205, 2339-2347 (2008).

31. Andrews, R. P., Kepley, C. L., Youssef, L., Wilson, B. S. \& Oliver, J. M. Regulation of the very late antigen-4-mediated adhesive activity of normal and nonreleaser basophils: roles for Src, Syk, and phosphatidylinositol 3-kinase. J. Leukoc. Biol. 70, 776-782 (2001).

32. Umemoto, E. et al. Nepmucin, a novel HEV sialomucin, mediates L-selectindependent lymphocyte rolling and promotes lymphocyte adhesion under flow. J. Exp. Med. 203, 1603-1614 (2006).

33. Mueller, H. et al. Tyrosine kinase Btk regulates E-selectin-mediated integrin activation and neutrophil recruitment by controlling phospholipase C (PLC) gamma2 and PI3Kgamma pathways. Blood 115, 3118-3127 (2010).

34. Rittirsch, D. et al. Cross-talk between TLR4 and FcgammaReceptorIII (CD16) pathways. PLoS Pathog. 5, e1000464 (2009).

35. Medvedev, A. E. et al. Role of TLR4 tyrosine phosphorylation in signal transduction and endotoxin tolerance. J. Biol. Chem. 282, 16042-16053 (2007).
36. Gilfillan, S., Ho, E. L., Cella, M., Yokoyama, W. M. \& Colonna, M. NKG2D recruits two distinct adapters to trigger NK cell activation and costimulation. Nat. Immunol. 3, 1150-1155 (2002).

37. Zhao, J. et al. IRF-8/interferon (IFN) consensus sequence-binding protein is involved in Toll-like receptor (TLR) signaling and contributes to the cross-talk between TLR and IFN-gamma signaling pathways. J. Biol. Chem. 281, 10073-10080 (2006).

38. Orabona, C. et al. Toward the identification of a tolerogenic signature in IDOcompetent dendritic cells. Blood 107, 2846-2854 (2006).

39. Takahashi, K. et al. Cooperative regulation of Fc receptor gamma-chain gene expression by multiple transcription factors, including Sp1, GABP, and Elf-1. J. Biol. Chem. 283, 15134-15141 (2008).

40. Cannon, J. P., O’Driscoll, M. \& Litman, G. W. Specific lipid recognition is a general feature of CD300 and TREM molecules. Immunogenetics 64, 39-47 (2012).

41. Gil-de-Gomez, L. et al. A phosphatidylinositol species acutely generated by activated macrophages regulates innate immune responses. J. Immunol. 190, 5169-5177 (2013).

42. Nakahashi-Oda, C. et al. Apoptotic cells suppress mast cell inflammatory responses via the CD300a immunoreceptor. J. Exp. Med. 209, 1493-1503 (2012).

43. Simhadri, V. R. et al. Human CD300a binds to phosphatidylethanolamine and phosphatidylserine, and modulates the phagocytosis of dead cells. Blood 119, 2799-2809 (2012).

44. Izawa, K. et al. The receptor LMIR3 negatively regulates mast cell activation and allergic responses by binding to extracellular ceramide. Immunity 37, 827-839 (2012)

45. Choi, S. C. et al. Cutting edge: mouse CD300f (CMRF-35-like molecule-1) recognizes outer membrane-exposed phosphatidylserine and can promote phagocytosis. J. Immunol. 187, 3483-3487 (2011).

46. Tian, L. et al. p85alpha recruitment by the CD300f phosphatidylserine receptor mediates apoptotic cell clearance required for autoimmunity suppression. Nat. Commun. 5, 3146 (2014).

47. Nakahashi-Oda, C., Tahara-Hanaoka, S., Honda, S., Shibuya, K. \& Shibuya, A. Identification of phosphatidylserine as a ligand for the CD300a immunoreceptor. Biochem. Biophys. Res. Commun. 417, 646-650 (2012).

48. Kaifu, T. et al. Osteopetrosis and thalamic hypomyelinosis with synaptic degeneration in DAP12-deficient mice. J. Clin. Invest. 111, 323-332 (2003).

49. Takai, T., Li, M., Sylvestre, D., Clynes, R. \& Ravetch, J. V. FcR gamma chain deletion results in pleiotrophic effector cell defects. Cell 76, 519-529 (1994).

50. Rittirsch, D., Huber-Lang, M. S., Flierl, M. A. \& Ward, P. A. Immunodesign of experimental sepsis by cecal ligation and puncture. Nat. Protoc. 4, 31-36 (2009).

51. Van Rooijen, N. The liposome-mediated macrophage 'suicide' technique. J. Immunol. Methods 124, 1-6 (1989).

\section{Acknowledgements}

We thank S. Mitsuishi and Y. Nomura for secretarial assistance, and the members of the laboratory for their discussions. This work was supported in part by grants provided by the Ministry of Education, Culture, Sports, Science and Technology of Japan.

\section{Author contributions}

N.T. conducted the experiments, analysed the data and wrote the paper. Y.-G.K. designed the experiments, analysed the data and wrote the paper. K.K., K.N. and E.U. performed the experiments and analysed the data, S.T.-H. and M.M. designed the experiments and analysed the data. C.N.-O. generated the AF251705 ${ }^{-/-}$mice and analysed the data. S.H. and K.S. analysed the data. A.S. supervised the overall project and wrote the paper.

\section{Additional information}

Supplementary Information accompanies this paper at http://www.nature.com/ naturecommunications

Competing financial interests: The authors declare no competing financial interests.

Reprints and permission information is available online at http://npg.nature.com/ reprintsandpermissions/

How to cite this article: Totsuka, N. et al. Toll-like receptor 4 and MAIR-II/CLM-4/ LMIR2 immunoreceptor regulate VLA-4-mediated inflammatory monocyte migration. Nat. Commun. 5:4710 doi: 10.1038/ncomms5710 (2014).

This work is licensed under a Creative Commons AttributionNonCommercial-NoDerivs 4.0 International License. The images or other third party material in this article are included in the article's Creative Commons license, unless indicated otherwise in the credit line; if the material is not included under the Creative Commons license, users will need to obtain permission from the license holder to reproduce the material. To view a copy of this license, visit http:/l creativecommons.org/licenses/by-nc-nd/4.0/ 